If two operators are restricted only by the two equations $\left(s_{1} s_{2}\right)^{\alpha_{1}} s_{1}=\left(s_{2} s_{1}\right)^{\beta_{1}} s_{2},\left(s_{1} s_{2}\right)^{\alpha_{1}-1} s_{1}=\left(s_{2} s_{1}\right)^{\beta_{1}+1} s_{2}$ they generate the dicyclic group of order $4\left(\alpha_{1}+\beta_{1}+1\right)$.

From the symmetry of the equations it results that the same group is generated by $s_{1}, s_{2}$ when they satisfy the two conditions $\left(s_{1} s_{2}\right)^{\alpha_{1}} s_{1}=\left(s_{2} s_{1}\right)^{\beta_{1}} s_{2},\left(s_{1} s_{2}\right)^{a_{1}+1} s_{1}=\left(s_{2} s_{1}\right)^{\beta_{1}-1} s_{2}$. The close contact of the present paper with the one entitled "Finite groups which may be defined by two operators satisfying two conditions $" *$ should be noted.

\title{
FUNDAMENTAL REGIONS FOR CYCLICAL GROUPS OF LINEAR FRACTIONAL TRANSFORMATIONS ON TWO COMPLEX VARIABLES.
}

\author{
BY PROFESSOR J. W. YOUNG.
}

(Read before the Southwestern Section of the American Mathematical Society, November 26,1910 .)

The purpose of this note is to call attention to a simple method for obtaining fundamental regions for cyclical groups of linear fractional transformations on two complex variables. The simplicity of the method is due to the fact that the determination of a fundamental region for a group of the specified kind is made to depend merely on the construction of such a region for a simply isomorphic group on a single complex variable. The method, moreover, may be readily extended to the case in which the number of variables is $n$, and to certain restricted types of groups which are not cyclical and not linear.

Let $V$ be any (non-identical) linear fractional transformation on two complex variables, and let $\Gamma$ be the cyclical group generated by $V$, i. e., consisting of all transformations $V^{n}(n=0$, $\pm 1, \pm 2, \cdots)$. We interpret $V$ as a collineation in a complex plane, and will consider separately each of the five types to which $V$ may belong according to the configuration of its invariant points and lines.

Suppose first that $V$ is of Type I, i. e., has three and only three invariant points forming the vertices of a triangle $A B C$. Every transformation of $\Gamma$ then has the same configuration of invariant elements. The group $\Gamma$ transforms the points on

* American Journal of Mathematics, vol. 31 (1909), p. 167. 
each of the sides of the invariant triangle according to a group of projective transformations on a single complex variable, the latter group being isomorphic with $\Gamma$. Moreover, it is evident that this isomorphism is certainly not multiple if the transfornations on the corresponding side of the triangle are not periodic. Suppose, then, that $V$ produces a transformation which is not elliptic (i. e., whose characteristic cross ratio is not of the form $e^{i \theta}, \theta$ real) on at least one side of the triangle $A B C$. Let $A B$ be such a side; the cyclical group on $A B$ is then either hyperbolic (non-involutoric) or loxodromic and is simply isomorphic with $\Gamma$. It is well known that any cyclical group of hyperbolic or loxodromic transformations on a single complex variable is properly discontinuous ; ${ }^{*}$ a familiar form of a corresponding fundamental region is bounded by two chains about the double points. $\dagger$

Let $R_{1}$ be a fundamental region for the cyclical group on $A B$, and let $R_{2}$ be the class of all points on all lines joining $C$ to points of $R_{1}$. $\quad R_{2}$ is then a fundamental region for the group $\Gamma$ if the lines $A C$ and $B C$ are regarded as singular. For if $P$ is any point of the complex plane not on a side of the triangle $A B C$, the line $P C$ meets $A B$ in a point $Q$. Since $R_{1}$ is a fundamental region for the group on $A B$, there exists in $\Gamma$ a transformation which transforms $Q$ into a point $Q^{\prime}$ of $R_{1}$. This transformation transforms $P$ into a point $P^{\prime}$ on the line $Q^{\prime} C$, i. e., into a point of $R_{2}$. Further, if $R_{2}$ contained two distinct interior points $P, P^{\prime}$ equivalent under a transformation of $\Gamma$, the lines $P C, P^{\prime} C$, which are necessarily distinct under the hypotheses made, would determine two distinct interior points of $R_{1}$ equivalent under the group on $A B$, which is impossible since $R_{1}$ is a fundamental region for the latter group.

\footnotetext{
* A group $G$ of transformations on the points of a space $S_{n}$ of $n$ (complex) dimensions is said to be properly discontinuous in $S_{n}$, provided there exists in $S_{n}$ a region $R_{n}$ of $n$ dimensions such that, (1) if $P$ is any point of $S_{n}$ not in $R_{n}$ (with the exception possibly of the points of certain "singular" spreads $S_{k}(k<n)$ in $\left.S_{n}\right)$, there exists in $G$ a transformation which transforms $P$ into a point of $R_{n}$; and $(2)$ two distinct interior points of $R_{n}$ are not equivalent under any transformation of $G$. For a more complete discussion of this notion the reader may consult, e. g., Fubini, Introduzione alla teoria dei gruppi discontinui e delle funzioni automorfe, Pisa, 1908, pp. $142 \mathrm{ff}$.

$\dagger$ If the points of the complex line $A B$ be interpreted in the usual way by the real points of a plane or sphere, the fundamental region in question is bounded by two circles about the double points (cf. Klein-Fricke, Elliptische Modulfunktionen, vol. 1, pp. $187 \mathrm{ff}$ ). We have used above the language of complex projective geometry, in which the totality of chains on a complex line correspond to the totality of real circles on the representing plane or sphere. Cf. a paper by the author, The geometry of chains on a complex line, Annals of Mathematics, 2d ser., vol. 11 (1909), pp. 33-48.
} 
There remains in the consideration of Type I only the case in which the characteristic cross ratio on each of the sides of the triangle $A B C$ is of the form $e^{i \theta}, \theta$ real. It is well known that a cyclical group of projectivities on a line generated by a projectivity whose cross ratio is $e^{i \theta}$ is properly discontinuous only if $\theta$ is commensurable with $\pi$. This is due to the fact that if the ratio $\theta: \pi$ is irrational, the corresponding group contains infinitesimal transformations, owing to the fact that under this hypothesis it is possible to determine two integers $m, n$ such that $|m \theta+n \cdot 2 \pi|<\epsilon$ for any positive $\epsilon$. Now it is readily seen that if $e^{i \theta_{1}}, e^{i \theta_{2}}, e^{i \theta_{3}}$ are the characteristic cross ratios on the sides of the triangle $A B C$, either $\theta_{1}, \theta_{2}, \theta_{3}$ are all commensurable with $\pi$, or else at least two of them are incommensurable with $\pi$. This follows directly from the theorem that the product of the three characteristic cross ratios taken in a proper way is equal to unity. If two of the $\theta^{\prime}$ 's, say $\theta_{1}, \theta_{2}$, are incommensurable with $\pi$, it follows that $\Gamma$ contains infinitesimal transformations. For it is then possible to determine three numbers $m, n, n^{\prime}$ such that the two relations $\left|m \theta_{1}+n \cdot 2 \pi\right|<\epsilon,\left|m \theta_{2}+n^{\prime} \cdot 2 \pi\right|<\epsilon$ hold simultaneously.* The corresponding transformation $V^{m}$ then clearly transforms any point $P$ into an arbitrarily near point $P^{\prime}$.

The only properly discontinuous groups of the type now under consideration are, therefore, those of finite order. If the cyclical group induced by such a $\Gamma$ on one of the sides of the invariant triangle is of the same order as $\Gamma$, the same method as that used above in the hyperbolic or loxodromic cases may evidently be employed to construct a fundamental region for $\Gamma$. This method fails, however, if the order of the group on each side of the invariant triangle is less than the order of $\Gamma . \dagger$

To obtain a fundamental region in this case we may proceed as follows: Let the order of the cyclical group on $A B$ be $m$, and suppose that the order of $\Gamma$ is greater than $m$. Let $R_{1}$ be a fundamental region of the group on $A B$, and let $R_{1}^{\prime}$ be a fundamental region of the group on $B C$ generated by $V^{m}$. Let $R_{2}$ be the class of all points in which a line joining $C$ to a point of $R_{1}$ meets a line joining $A$ to a point of $R_{1}^{\prime}$. The region $R_{2}$ is then a fundamental region for $\Gamma$. For let $P$ be any point of the complex plane not on a side of the triangle $A B C$,

\footnotetext{
* For an elementary proof of this fact, and its generalization to $\boldsymbol{n}$ irrational ratios, cf. Minkowski, Diophantische Approximationen, p. 7.

+ This could happen, for example, if the orders of the groups on the sides of $A B C$ were respectively 15,10 , and 6 ( since $\left.\frac{1}{15}+\frac{1}{10}-\frac{1}{6}=0\right)$.
} 
and let the line $P C$ meet $A B$ in $Q$. There then exists in $\Gamma$ a transformation $V^{a}$ which transforms $Q$ into a point $Q^{\prime}$ of $R_{1}$. The point $P$ is transformed by $V^{a}$ into a point of the line $Q^{\prime} C$. If $P^{\prime}$ is not a point of $R_{2}$, let the line $P^{\prime} A$ meet $B C$ in $S$. There then exists a transformation of the cyclical group generated by $V^{m}$, i. e. a transformation $V^{m b}$, which transforms $S$ into a point $S^{\prime}$ of $R_{1}^{\prime}$. This transformation will transform $P^{\prime}$ in to the intersection $P^{\prime \prime}$ of $Q^{\prime} C$ and $S^{\prime} A$, since $V^{m b}$ leaves the line $Q^{\prime} C$ invariant. But $P^{\prime \prime}$ is by definition a point of $R_{2}$. On the other hand, suppose $R_{2}$ contained two interior points $P$, $P^{\prime}$ which are equivalent under a transformation $V^{e}$ of $\Gamma$. If the lines $P C, P^{\prime} C$ are distinct, the hypothesis would imply the existence of two distinct interior points of $R_{1}$ equivalent under $\Gamma$, which contradicts the assumption that $R_{1}$ is a fundamental region of the group on $A B$. If, however, $P, P^{\prime}$ are collinear with $C$, the lines $P A, P^{\prime} A$ are distinct. Since the transformation transforming $P$ into $P^{\prime}$ now leaves $P C$ invariant, it is of the form $V^{k m}$; but this would imply the existence in $R_{1}^{\prime}$ of two points equivalent under a transformation of this form, which again contradicts the assumption regarding $R_{1}^{\prime}$. This completes the proof that $R_{2}$ is a fundamental region for $\Gamma$.

The points on two of the sides of the invariant triangle have in the above discussion been left out of account; i. e., we have regarded the lines in question as singular. It is clear, however, that if the groups generated on such an invariant line are properly discontinuous, we need only add to $R_{2}$ a fundamental region for every such group on an invariant line in order to have a region which is fundamental for all points which are transformed in a properly discontinuous way.

The discussion of the remaining four types now offers no difficulty as the first method described above will readily apply. This method may be described in slightly different language as follows: The transformation $V$ transforms the lines through any invariant point according to a group isomorphic with $\Gamma$. If this isomorphism is simple, we choose a fundamental region of lines for the group on the invariant point. The region consisting of all the points on these lines is a fundamental region for $\Gamma$. If $V$ is of Type II, i. e., has two and only two invariant points, the group on one of these points is parabolic, and hence (since it is not of finite order) is simply isomorphic with $\Gamma$. If $V$ is of Type III, i. e., has one and only one invariant point, the group on this point is likewise simply isomorphic with $\Gamma$. If 
$V$ is of Type IV (a homology) the group induced by $\Gamma$ on any point of the axis of homology is simply isomorphic with $\Gamma$ (it may not be properly discontinuous, however, in which case $\Gamma$ has no fundamental region). Finally, if $V$ is of Type $V$ (an elation), the induced group on any point of the axis distinct from the center is parabolic and simply isomorphic with $\Gamma$. The method then suffices to determine a fundamental region for every properly discontinuous cyclical group of linear fractional transformations on two complex variables.

It is readily seen, moreover, that the method may be extended to the case where the number of variables is $n$. It will serve also to simplify the problem of determining a fundamental region of any properly discontinuous group of transformations on the points of an $S_{n}$ which leaves a point of $S_{n}$ invariant, provided the group on the $S_{n-k}$ (for some $k=1,2, \cdots, n-1$ ) through this invariant point is simply isomorphic with the given group. The simplification consists in reducing the problem to the determination of a fundamental region for a simply isomorphic group on a smaller number (namely, $k$ ) of variables.

LAWRENCE, KAN., November, 1910 .

\section{ON THE RELATIVE DISCRIMINANT OF A CERTAIN KUMMER FIELD.}

BY PROFESSOR JACOB WESTLUND.

(Read before the American Mathematical Society, September 7, 1910.)

Is a paper published in the Transactions of the American Mathematical Society for October, 1910, I determined the fundamental number or discriminant of the algebraic number field $k(\sqrt[p]{m})$, generated by the real $p$ th root of the positive integer $m$. Denoting this discriminant by $d$ and setting $m=a_{1} a_{2}^{2} \ldots a_{p-1}^{p-1}$, where $a_{1} a_{2} \ldots a_{p-1}$ is not divisible by the square of a prime, the following result was obtained :

$$
\begin{aligned}
& d=(-1)^{(p-1) / 2} p^{p-2}\left(a_{1} a_{2} \cdots a_{p-1}\right)^{p-1} \\
& d=(-1)^{(p-1) / 2} p^{p}\left(a_{1} a_{2} \cdots a_{p-1}\right)^{p-1},
\end{aligned}
$$

according as $b^{p-1}-a_{p-1}^{p-1}$ is divisible by $p^{2}$ or not, where $b=$ $a_{1} a_{2}^{2} \ldots a_{p-2}^{p-2}$. 